\title{
Anisotropic character of the metal-to-metal transition in $\operatorname{Pr}_{4} \mathrm{Ni}_{3} \mathrm{O}_{10}$
}

\author{
Shangxiong Huangfu, ${ }^{1}$ Gawryluk Dariusz Jakub $\odot,{ }^{2}$ Xiaofu Zhang $\odot,{ }^{1}$ Olivier Blacque, ${ }^{3}$ Pascal Puphal $\odot,{ }^{2}$ \\ Ekaterina Pomjakushina, ${ }^{2}$ Fabian O. von Rohr, ${ }^{3}$ and Andreas Schilling ${ }^{1}{ }^{1}$ \\ ${ }^{1}$ Department of Physics, University of Zurich, Winterthurerstrasse 190 CH-8057 Zurich Switzerland \\ ${ }^{2}$ Laboratory for Multiscale Materials Experiments (LMX), Paul Scherrer Institute (PSI), \\ Forschungsstrasse 111, CH-5232 Villigen, Switzerland \\ ${ }^{3}$ Department of Chemistry, University of Zurich, Winterthurerstrasse 190 CH-8057 Zurich Switzerland
}

(Received 16 January 2020; revised manuscript received 21 February 2020; accepted 27 February 2020; published 25 March 2020)

\begin{abstract}
As a member of the Ruddlesden-Popper $L n_{n+1} \mathrm{Ni}_{\mathrm{n}} \mathrm{O}_{3 \mathrm{n}+1}$ series rare-earth-nickelates, $\mathrm{Pr}_{4} \mathrm{Ni}_{3} \mathrm{O}_{10}$ consists of infinite quasi-two-dimensional perovskite-like Ni-O based layers. Although a metal-to-metal phase transition at $T_{p t} \approx 157 \mathrm{~K}$ has been revealed by previous studies, a comprehensive study of physical properties associated with this transition has not yet been performed. We have grown single crystals of $\mathrm{Pr}_{4} \mathrm{Ni}_{3} \mathrm{O}_{10}$ at high oxygen pressure, and report on the physical properties around that phase transition, such as heat-capacity, electrictransport, and magnetization. We observe a distinctly anisotropic behavior between in-plane and out-of-plane properties: a metal-to-metal transition at $T_{p t}$ within the $a-b$ plane, and a metal-to-insulator-like transition along the $c$ axis with decreasing temperature. Moreover, an anisotropic and anomalous negative magnetoresistance is observed at $T_{p t}$ that we attribute to a slight suppression of the first-order transition with magnetic field. The magnetic susceptibility can be well described by a Curie-Weiss law, with different Curie constants and Pauli-spin susceptibilities between the high-temperature and the low-temperature phases. The single crystal x-ray diffraction measurements show a shape variation of the different $\mathrm{NiO}_{6}$ octahedra from the high-temperature phase to the low-temperature phase. This subtle change of the environment of the Ni sites is likely responsible for the different physical properties at high and low temperatures.
\end{abstract}

DOI: 10.1103/PhysRevB.101.104104

\section{INTRODUCTION}

Quasi-two-dimensional (quasi-2D) transition-metal oxides generally show a variety of intriguing electronic properties due to their strongly correlated $d$-electrons $[1,2]$, electric anisotropy [3-6], as well as the mixed-valence states of the transition-metal ions $[7,8]$. Such layered compounds can display high-temperature superconductivity $[9,10]$, colossal magnetoresistance (MR) [11,12], charge stripes [13,14], and other complex physical phenomena. There exist various transitions between different phases with distinct properties, e.g., temperature or pressure induced metal-to-insulator transitions [15-19], or a doping induced Mott-insulator to superconductor transition in the cuprates $[9,20,21]$. The RuddlesdenPopper (R-P) rare-earth nickelates $L n_{n+1} \mathrm{Ni}_{n} \mathrm{O}_{3 \mathrm{n}+1}(L n=$ rare earth) have recently attracted a lot of interest because they contain quasi-2D Ni-O layers which are similar to the $\mathrm{Cu}-\mathrm{O}$ layers in the high-temperature superconductors [22-25], and superconductivity has indeed been reported to occur in a structurally and electronically closely related compound $\mathrm{Nd}_{0.8} \mathrm{Sr}_{0.2} \mathrm{NiO}_{2}$ with planar Ni-O layers [26]. The layers in $L n_{n+1} \mathrm{Ni}_{n} \mathrm{O}_{3 n+1}$ are piled up to an infinite perovskite like layered structure. They are connected by $\mathrm{NiO}_{6}$ octahedra via shared vertex (both equatorial and axial) oxygen ions, and separated by rock-salt like layers $(L n-\mathrm{O})$ along the crystallographic $c$ axis.

These rare earth nickelates have shown various phases at different temperatures and magnetic fields. To be specific, perovskite $L n \mathrm{NiO}_{3}(L n=\mathrm{Y}$, Pr to Gd, Dy to $\mathrm{Lu}, n=\infty$ ) show a metal-to-insulator phase transition [16,27,28], which is accompanied by structural changes [29]. Close to the transition temperature, the pronounced electron-lattice interaction induces a distortion of the $\mathrm{NiO}_{6}$ octahedra, leading to significant changes of the Ni-O bond lengths at different crystallographic $\mathrm{Ni}$ sites in the low-temperature phases. Ultimately, it is a charge disproportionation commensurate with the crystallographic unit cell that generates this phase transition [30-33]. Similarly, in the $\mathrm{Ln}_{2} \mathrm{NiO}_{4}$ series $(n=1)$, a first-order structural phase transition occurs in $\mathrm{La}_{2} \mathrm{NiO}_{4}$, $\mathrm{Pr}_{2} \mathrm{NiO}_{4}$, and $\mathrm{Nd}_{2} \mathrm{NiO}_{4}$, from a high-temperature orthorhombic phase to a low-temperature tetragonal phase at around $80 \mathrm{~K}$ [34], $115 \mathrm{~K}$ [35], and $130 \mathrm{~K}$ [36,37], respectively. By contrast, the only known compound of the $\mathrm{Ln}_{3} \mathrm{Ni}_{2} \mathrm{O}_{7}(n=2)$ series, $\mathrm{La}_{3} \mathrm{Ni}_{2} \mathrm{O}_{7}$, shows a structural transition at $550 \mathrm{~K}$ [38]. However, it exhibits a metallic behavior with a weak "kink" around $128 \mathrm{~K}$ in the resistivity, and a downward "peak" in the magnetization near $128 \mathrm{~K}$ [39]. In the $\mathrm{Ln}_{4} \mathrm{Ni}_{3} \mathrm{O}_{10}$ series ( $L n=\mathrm{La}$, Pr and $\mathrm{Nd}$, with $n=3$ ), analogous indications for transitions have been reported in terms of a resistivity shift in the temperature range of 140-165 K, and a slightly anomalous magnetization in the same temperature range in $\mathrm{La}_{4} \mathrm{Ni}_{3} \mathrm{O}_{10}$ [24,39-41]. Nonetheless, a comprehensive study of the physical properties near these transitions in the $L n_{4} \mathrm{Ni}_{3} \mathrm{O}_{10}$ series is still lacking.

The significant in-plane and out-of-plane anisotropy in most R-P rare-earth nickelates leads to quasi-2D electronic 
and magnetic properties $[42,43]$. Due to the high melting point of nickelates and their complex Ni valence states, the growth of corresponding single crystals is rather difficult [44], and generally necessitates the application of high oxygen pressure. This makes the study of the anisotropic properties of this compound series very difficult. Although the successful single-crystal growth of $\mathrm{LaNiO}_{3}$ has recently been reported, anisotropic properties are not expected in this compound with an isotropy in its crystal structure along the three pseudo-cubic directions [45,46]. Single crystals of $\mathrm{Ln}_{2} \mathrm{NiO}_{4}(\mathrm{Ln}=\mathrm{La}$, Pr, $\mathrm{Nd}$ ) have been synthesized by the skull-melting method [47], and the electric-transport measurements on $\mathrm{La}_{2} \mathrm{NiO}_{4}$ showed that the in-plane resistivity is three orders of magnitude smaller than the out-of-plane resistivity [48]. Single crystals of $\mathrm{La}_{4} \mathrm{Ni}_{3} \mathrm{O}_{10}$ and $\mathrm{Pr}_{4} \mathrm{Ni}_{3} \mathrm{O}_{10}$ have also been obtained recently $[24,49]$, and angle-resolved photoemission spectroscopy measurements on $\mathrm{La}_{4} \mathrm{Ni}_{3} \mathrm{O}_{10}$ show a gap opening at a phase transition, which is likely associated with the occurrence of a charge-density wave, but no anisotropic properties have been reported so far [24].

In this report, we have successfully grown $\mathrm{Pr}_{4} \mathrm{Ni}_{3} \mathrm{O}_{10}$ single crystals in a traveling solvent floating-zone furnace at high oxygen pressure. We have performed measurements on the crystal structure, the heat capacity, and the electric and magnetic properties, which all exhibit distinct changes at the phase transition temperature at $T_{p t} \approx 157 \mathrm{~K}$. We show that this transition is slightly field-dependent and of first order.

\section{METHODS}

We first synthesized $\mathrm{Pr}_{4} \mathrm{Ni}_{3} \mathrm{O}_{10}$ powder by a sol-gel method with citric acid assistance to obtain a pure phase at relatively moderate reaction conditions [50], and the precursor powder was annealed at $1100{ }^{\circ} \mathrm{C}$ in oxygen atmosphere for $24 \mathrm{~h}$. The single-crystal growth of $\mathrm{Pr}_{4} \mathrm{Ni}_{3} \mathrm{O}_{10}$ was performed in an optical-image traveling solvent floating-zone furnace in an oxygen atmosphere at a pressure of $\approx 140$ bar. The obtained single crystals are as large as $3.3 \times 1.6 \times 0.3 \mathrm{~mm}^{3}$ [see the inset of Fig. 1(c)]. The details concerning the synthesis procedures are described in the Supplemental Material [51-59]. The structural changes of the $\mathrm{Pr}_{4} \mathrm{Ni}_{3} \mathrm{O}_{10}$ single crystals were characterized by single crystal $\mathrm{x}$-ray diffraction (XRD) both at room temperature and at $150 \mathrm{~K}$. An XRD measurement on pulverized single-crystal powder shows sharp peaks [Fig. 1(c)] revealing the high sample quality from the reactions.

The electrical resistivity was measured with a Physical Property Measurement System (PPMS, Quantum Design Inc.) and a standard four-probe technique with electrical contacts attached with silver paste. The zero-field transport measurements were performed for both in-plane and out-of-plane configurations in the temperature range from 10 to $300 \mathrm{~K}$. The magnetotransport measurements were done in external magnetic fields up to $B=9 \mathrm{~T}$, where $B$ was oriented perpendicular to the current. The heat-capacity was measured with the heat-capacity option of the PPMS in the temperature range between 10 and $300 \mathrm{~K}$ in zero field and in $B=7 \mathrm{~T}$. The magnetic properties were studied in a Magnetic Properties Measurement System (MPMS 3 from Quantum Design Inc.),
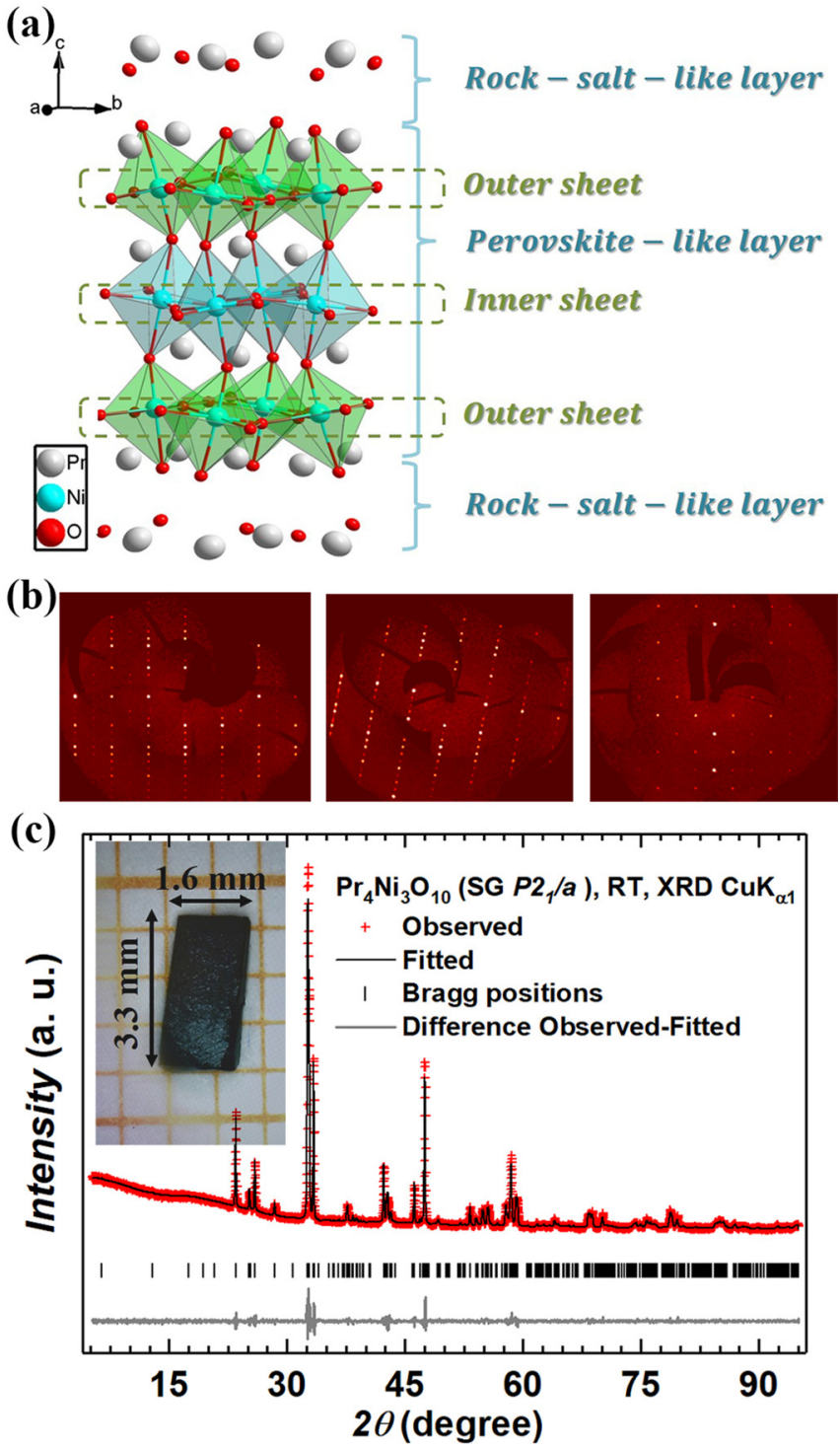

FIG. 1. (a) Illustration of the layered crystal structure of $\mathrm{Pr}_{4} \mathrm{Ni}_{3} \mathrm{O}_{10}$; (b) $(O k l),(h O k)$ and $(h k 0)$ planes (from left to right) of single crystal $\mathrm{Pr}_{4} \mathrm{Ni}_{3} \mathrm{O}_{10}$ based on a single crystal diffraction experiment performed at $150(1) \mathrm{K}$ ( $P 2_{1} / a$ settings); (c) Experimental powder XRD $\left(\mathrm{Cu} K_{\alpha 1}\right.$ radiation) pattern (red crosses) for pulverized single crystal sample of $\mathrm{Pr}_{4} \mathrm{Ni}_{3} \mathrm{O}_{10}$ at room temperature. The black line corresponds to the best fit from the Rietveld refinement analysis. Lower vertical marks denote the Bragg peak positions. The bottom, grey line represents the difference between experimental and calculated points; the inset of (c) shows the image of the crystal used for all the measurements.

which is equipped with a reciprocating sample option, with external fields $B$ both parallel and perpendicular to the $c$ axis.

\section{RESULTS}

In $\mathrm{Pr}_{4} \mathrm{Ni}_{3} \mathrm{O}_{10}$, featuring $P 2_{1} / a$ space group, there exist two different types of $\mathrm{Ni}$ sites in the crystal structure as it is illustrated in Fig. 1(a), representing the $\mathrm{Ni}$ sites in the inner perovskite layer $\left(\mathrm{Ni}_{\text {in }}\right)$ and in the outer perovskite layers $\left(\mathrm{Ni}_{\text {out }}\right)$, respectively. All the $\mathrm{Ni}$ ions are located in the center 
TABLE I. Average Ni-O bond lengths at different $\mathrm{Ni}$ sites at different temperatures. $\mathrm{e}=$ equatorial and $\mathrm{a}=$ axial.

\begin{tabular}{lccccc}
\hline \hline & \multicolumn{2}{c}{ Room temperature $\left(T>T_{p t}\right)$} & & \multicolumn{2}{c}{$150 \mathrm{~K}\left(T<T_{p t}\right)$} \\
\cline { 2 - 3 } \cline { 5 - 6 } & $\left\langle\mathrm{Ni}-\mathrm{O}_{\mathrm{e}}\right\rangle(\AA)$ & $\left\langle\mathrm{Ni}-\mathrm{O}_{\mathrm{a}}\right\rangle(\AA)$ & & $\left\langle\mathrm{Ni}-\mathrm{O}_{\mathrm{e}}\right\rangle(\AA)$ & $\left\langle\mathrm{Ni}-\mathrm{O}_{\mathrm{a}}\right\rangle(\AA)$ \\
\hline $\mathrm{Ni}_{\text {in }}$ & 1.94 & 1.92 & & 1.93 & 1.90 \\
$\mathrm{Ni}_{\text {out }}$ & 1.93 & 2.08 & & 1.93 & 2.08 \\
\hline \hline
\end{tabular}

of the disordered $\mathrm{NiO}_{6}$ octahedra, resulting in a monoclinic symmetry of the unit cell. It has been recently shown that upon lowering the temperature, the $b$ axis was expanded below $T_{p t}$, giving rise to a structural change at this transition temperature [49]. We, therefore, performed single-crystal XRD both at room temperature (above $T_{p t}$ ) and at $150 \mathrm{~K}$ (below $T_{p t}$ ), the detailed results of which are listed in the Supplemental Material [51,55-59]. We verify a larger $b / c$ value at $150 \mathrm{~K}$ than at room temperature, which is consistent with the previous results [49]. Furthermore, the bond lengths of Ni-O show distinctly different temperature dependences at the different
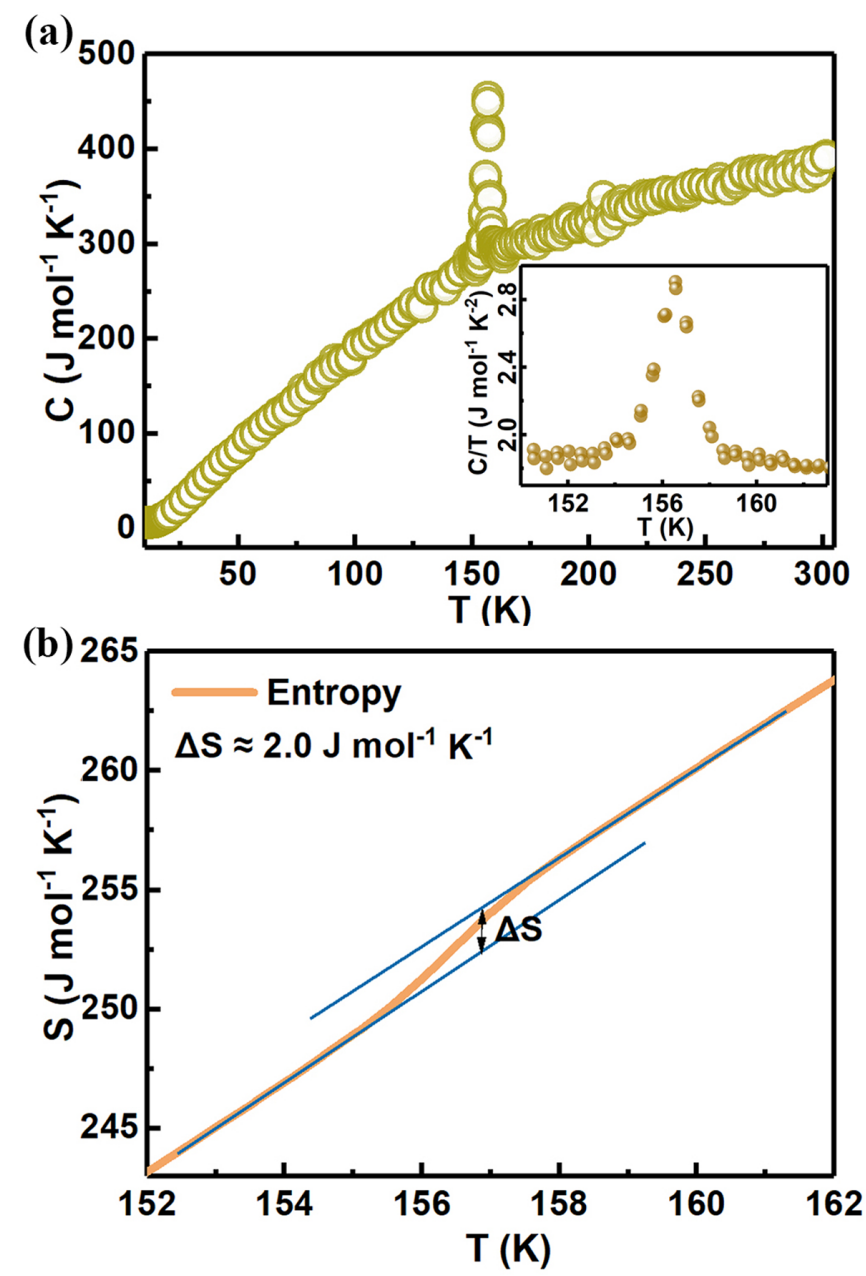

FIG. 2. (a) Temperature dependence of heat-capacity of $\mathrm{Pr}_{4} \mathrm{Ni}_{3} \mathrm{O}_{10}$ between 10 and $300 \mathrm{~K}$. Inset: $C / T$ as a function of temperature around $T_{p t}$; (b) Corresponding entropy $S(T)$, showing a steplike feature at $T_{p t}$.

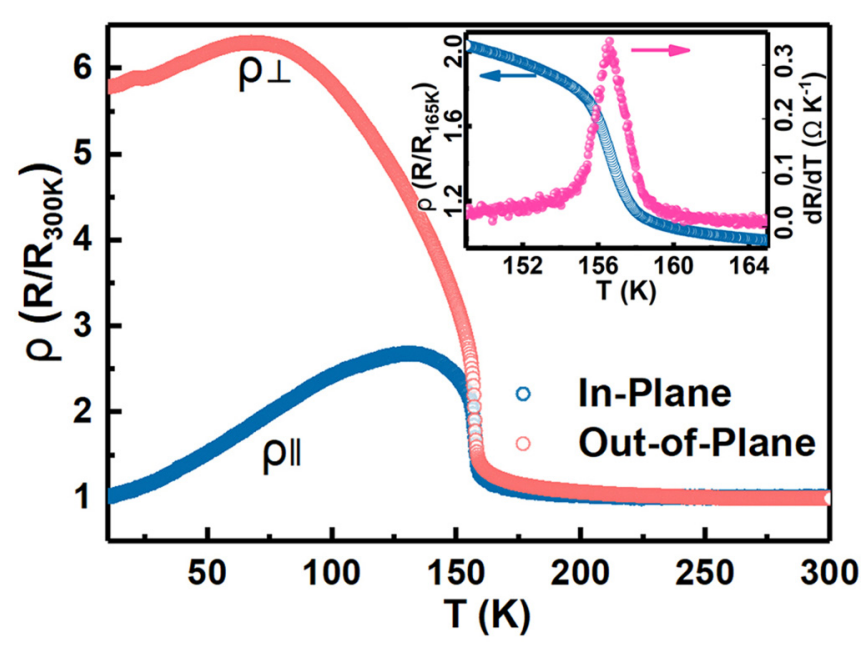

FIG. 3. Temperature dependence of the normalized zero-field resistivities of $\operatorname{Pr}_{4} \mathrm{Ni}_{3} \mathrm{O}_{10}$, both in-plane $\left(\rho_{\|}\right)$and out-of-plane $\left(\rho_{\perp}\right)$; the inset shows resistivity data $\rho_{\|}$around $T_{p t}$ of both the normalized resistivity and its derivative, as functions of temperature.

Ni sites, which we summarized in Table I. To be specific, the $\mathrm{Ni}_{\text {in }}-\mathrm{O}$ lengths clearly change between room temperature and $150 \mathrm{~K}$, while there are no visible differences between the corresponding $\mathrm{Ni}_{\text {out }}-\mathrm{O}$ bond lengths. We conclude that the inner $\mathrm{NiO}_{6}$ octahedra shrink during the phase transition, while the outer $\mathrm{NiO}_{6}$ octahedra remain almost unaffected.

Figure 2(a) shows the temperature dependence of the heat capacity $C$ of the $\mathrm{Pr}_{4} \mathrm{Ni}_{3} \mathrm{O}_{10}$ single crystal in zero magnetic field between 10 and $300 \mathrm{~K}$. The heat capacity reaches $\approx 390 \mathrm{~J} \mathrm{~mol}^{-1} \mathrm{~K}^{-1}$ at $300 \mathrm{~K}$, approaching the classical value from the Dulong-Petit model $\left(C=3 n R \approx 424 \mathrm{~J} \mathrm{~mol}^{-1} \mathrm{~K}^{-1}\right.$, where $R=8.314 \mathrm{~J} \mathrm{~mol}^{-1} \mathrm{~K}^{-1}$ is the gas constant and $n$ is the number of atoms per formula unit). A clear $\delta$-like peak appears at $T_{p t} \approx 157 \mathrm{~K}$, with a peak height in $C / T$ of $\approx 1.05 \mathrm{~J} \mathrm{~mol}^{-1} \mathrm{~K}^{-2}$ and a peak width of $\approx 4 \mathrm{~K}$ [see inset of Fig. 2(a)], which is considerably sharper than previously reported results [49]. By integrating $C / T$ vs $T$, we obtain the entropy $S(T)$ that displays an abrupt change $(\Delta S \approx$ $2.0 \mathrm{~J} \mathrm{~mol}^{-1} \mathrm{~K}^{-1}$ ) at the phase transition [Fig. 2(b)], indicating a possible first-order phase transition. We note, however, that there is no detectable hysteresis around $T_{p t}$ within the temperature resolution of the measurement $(\approx 0.5 \mathrm{~K})$ (Fig. S1), and that a value of $\Delta S$ lower than $R \ln (2)$ is often observed at phase transition in metallic nickel oxides [60].

Considering the structural change at $T_{p t}$ [49] as it is revealed by our single crystal XRD measurements, we may expect to a variety of further changes of the physical properties of $\mathrm{Pr}_{4} \mathrm{Ni}_{3} \mathrm{O}_{10}$ as we shall see below.

To clarify the influence of this phase transition on the electric-transport properties, we measured the temperaturedependent in-plane $\left(\rho_{\|}\right)$and out-of-plane $\left(\rho_{\perp}\right)$ resistivities in zero magnetic field. Figure 3 shows the temperature dependence of $\rho_{\|}$and $\rho_{\perp}$ from 10 to $300 \mathrm{~K}$, normalized to the corresponding resistivities at $300 \mathrm{~K}$. The absolute values at room temperature are roughly $\rho_{\|} \approx 5.6 \times 10^{-3} \Omega \mathrm{cm}$ and $\rho_{\perp} \approx 0.047 \Omega \mathrm{cm}$, respectively, which is considerably less than reported for the oxygen-reduced single crystals $\mathrm{Pr}_{4} \mathrm{Ni}_{3} \mathrm{O}_{8}$ $(\approx 67 \Omega \mathrm{cm})$ [25], but is in line with data published for 

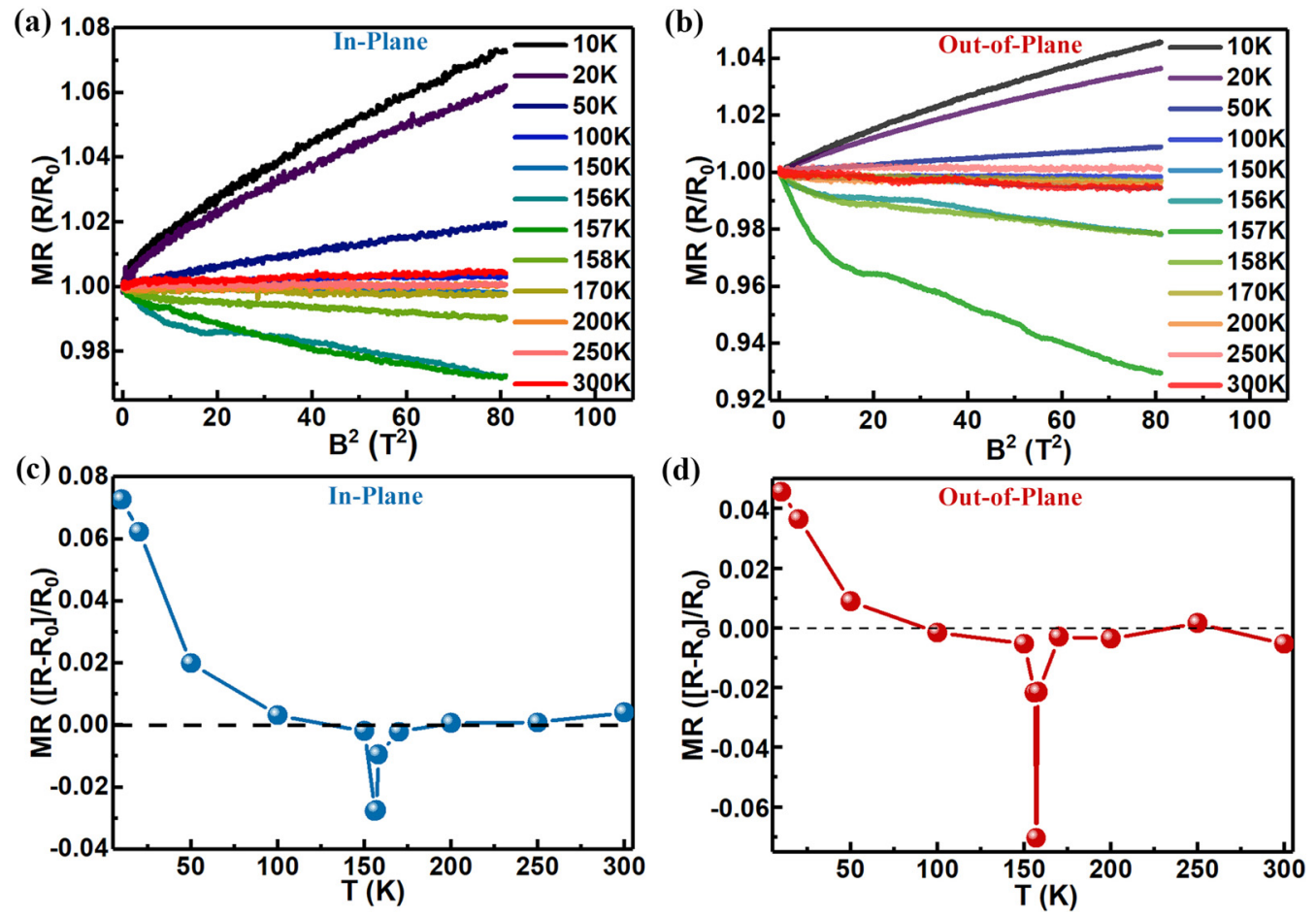

FIG. 4. The magnetoresistivity measurements of $\operatorname{Pr}_{4} \mathrm{Ni}_{3} \mathrm{O}_{10}$ for both in-plane (a) and out-of-plane (b) configurations, plotted vs. $B^{2}$ for clarity; (c) and (d) show the respective temperature dependences of the magnetoresistivities for $B=9 \mathrm{~T}$.

polycrystalline $\mathrm{Pr}_{4} \mathrm{Ni}_{3} \mathrm{O}_{10}(\approx 0.015 \Omega \mathrm{cm})$ [40]. The ratio of the measured values at room temperature is $\rho_{\perp} / \rho_{\|} \approx 8.4$. In the high-temperature regime, these quantities show a similar metallic behavior. With decreasing temperature, however, both $\rho_{\|}(T)$ and $\rho_{\perp}(T)$ exhibit a sharp increase but a qualitatively different behavior below $T_{p t}$. Within the $a-b$ plane, the resistivity $\rho_{\|}(T)$ shows a metal-to-metal transition, while along the $c$ axis, the $\rho_{\perp}(T)$ significantly increases with decreasing temperature over a broad temperature range, indicating rather a metal-to-insulator-like transition. We cannot rule out that the fact that $\rho_{\perp}(T)$ appears to decrease again in the low-temperature limit is due to a misalignment of the electrical leads and the irregular shape of the thin crystal, thereby mixing $\rho_{\|}$and $\rho_{\perp}$ in the measured data to some extent. Therefore, the room-temperature ratio $\rho_{\perp} / \rho_{\|} \approx$ 8.4 can only be taken as a lower bound of the resistance anisotropy.

Since the magnetotransport properties are closely related to the electronic structure near the Fermi surface, we also measured the MR along different directions between 10 and $300 \mathrm{~K}$ in detail. In Figs. 4(a) and 4(b) we show the resistance $R(B)$ for varying external magnetic field at constant temperatures $T$, normalized to the respective zero-field values $R(0)$. The response to the magnetic field is positive at low temperatures (with $\partial \rho / \partial B>0$ ) for both in-plane and out-ofplane configurations, and becomes weaker with increasing temperature, which is very common for simple metals $[61,62]$. In a very narrow region around the phase transition at $T_{p t}$, however, the MR effect becomes large and negative, i.e., with $\partial \rho / \partial B<0$. In the Figs. 4(c) and 4(d) we are plotting the relative changes in resistance between zero field and $B=$ $9 \mathrm{~T}$, indicating a seemingly anomalous sign change around
$T_{\mathrm{pt}}$. Whereas outside this transition region, the normalized MR of the in-plane resistance $\rho_{\|}$is larger than that of the out-of-plane resistance $\rho_{\perp}$, the negative MR around $T_{\mathrm{pt}}$ is larger for perpendicular current transport.

The R-P $\mathrm{Ln}_{4} \mathrm{Ni}_{3} \mathrm{O}_{10}$ nickelates have been reported to show a rather complicated temperature dependence of the magnetic behavior $[42,63]$. In Figs. 5(a) and 5(b) we present the magnetic susceptibility near $T_{p t}$ for $B \| c$ and $B \perp c$, showing a steplike feature at $T_{p t}$, which is less obvious when compared with the $\mathrm{La}_{4} \mathrm{Ni}_{3} \mathrm{O}_{10}$ peer $[39,63]$, however. The temperature derivative of $M(T)$ allows for a clearer identification of the transition temperature [Figs. 5(a) and 5(b)]. The magnetic susceptibility for zero-field-cooling (ZFC) and field-cooling (FC) overlap nearly in the whole measurement range (Fig. S2), without any detectable hysteretic behavior. The temperature dependence of the magnetic susceptibility on both sides of the transition can be well fitted to a Néeltype law, $\chi(T)=\frac{C}{T+\Theta}+\chi_{0}$ (results are listed in Table II), demonstrating the paramagnetic nature of $\operatorname{Pr}_{4} \mathrm{Ni}_{3} \mathrm{O}_{10}$ in the whole measured temperature range. As expected, the resulting Curie constants $C$ on both sides of the transition are nearly field independent and isotropic (see Table II and Fig. S3). In the low-temperature phase, the Curie constant is $C \approx 6.4 \mathrm{emu} \mathrm{K} \mathrm{Oe}^{-1} \mathrm{~mol}^{-1}$, which is compatible with the assumption that the magnetization in this temperature range is governed by the $\operatorname{Pr}^{3+}$ ions with a magnetic moment of $3.58 \mu_{\mathrm{B}}$ per $\operatorname{Pr}^{3+}$, i.e., the theoretical value for free $\operatorname{Pr}^{3+}$ ions. Similar values have been reported for $\mathrm{PrNiO}_{3}\left(3.31 \mu_{\mathrm{B}}\right)$ [64] and $\mathrm{Pr}_{2} \mathrm{NiO}_{4}\left(3.73 \mu_{\mathrm{B}}\right)$ [65]. The $\mathrm{Ni}$ ions do not seem to significantly contribute to the magnetic susceptibility at low temperatures. In the high-temperature phase, the resulting Curie constant $\left(C \approx 7.6 \mathrm{emu} \mathrm{K} \mathrm{Oe}^{-1} \mathrm{~mol}^{-1}\right)$ is $\approx 18 \%$ larger, 

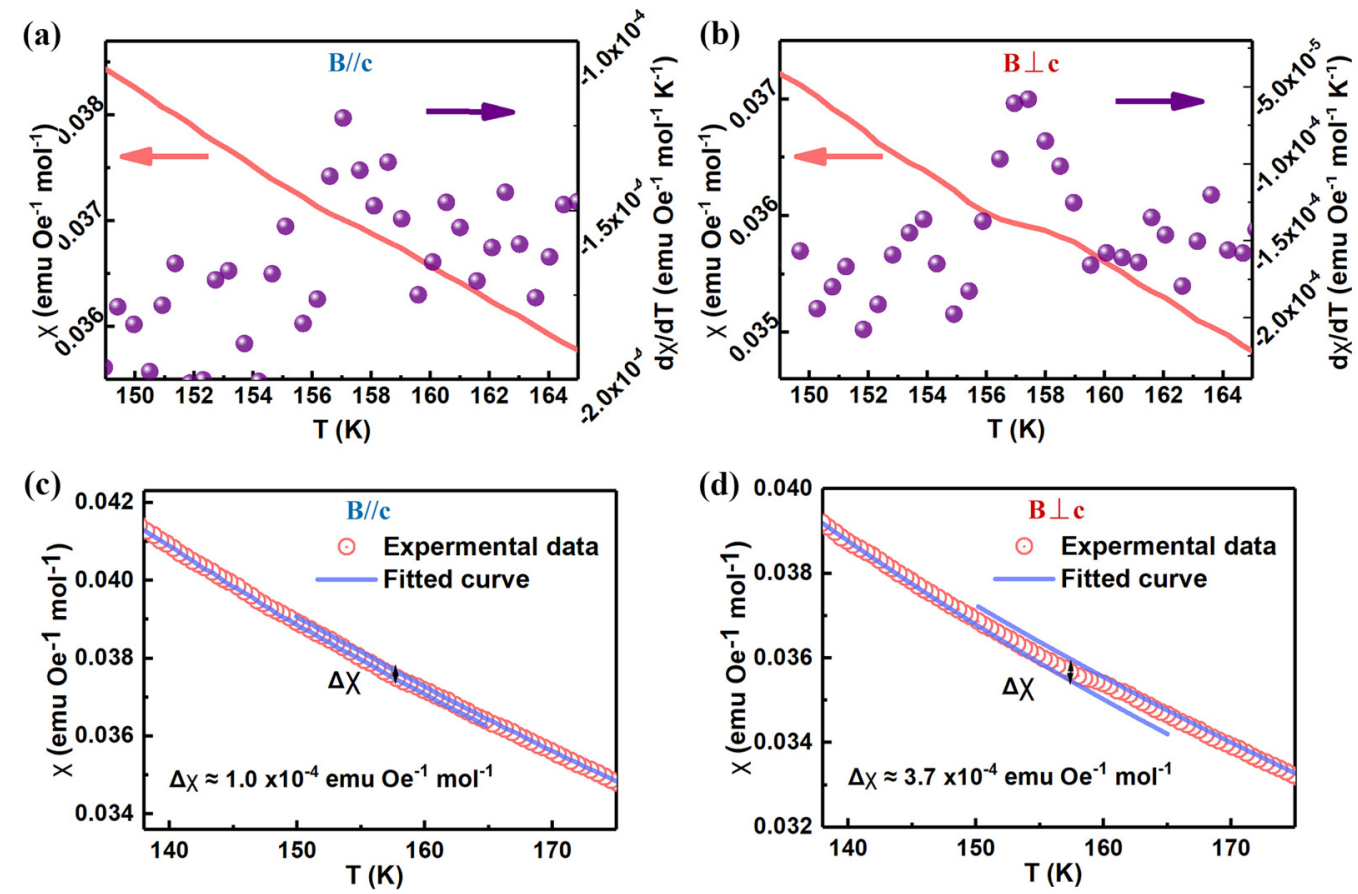

FIG. 5. Field-cooling (FC) magnetic susceptibility of $\mathrm{Pr}_{4} \mathrm{Ni}_{3} \mathrm{O}_{10}$ and its derivative in an external magnetic field $B=0.1 \mathrm{~T}$ near the phase transition for $B \| c$ (a) and $B \perp c$ (b); Magnetic-susceptibility data with Néel-type fits above and below $T_{p t}$ for $B \| c$ (c) and $B \perp c$ (d); $\Delta \chi$ corresponds to the steplike increase of the magnetic susceptibility from the low-temperature to the high-temperature phase; the corresponding zero-field cooling (ZFC) data overlap with no detectable hysteresis and are therefore not shown for clarity.

indicating the presence of an additional contribution from the $\mathrm{Ni}$ ions to the magnetization.

The fitted values for the Pauli paramagnetic susceptibility are quite large in the low-temperature phase, with $\chi_{0}^{L} \approx 3.9 \times 10^{-3} \mathrm{emu} \mathrm{Oe}^{-1} \mathrm{~mol}^{-1}$ for $B \| c$ and $\chi_{0}^{L} \approx$ $2.2 \times 10^{-3} \mathrm{emu} \mathrm{Oe}^{-1} \mathrm{~mol}^{-1}$ for $B \perp c$, while they are substantially smaller at high temperatures above $T_{p t}$, with $\chi_{0}^{H} \approx 9 \times 10^{-4} \mathrm{emu} \mathrm{Oe}^{-1} \mathrm{~mol}^{-1}$ for $B \| c$ and $\chi_{0}^{H} \approx 2.5 \times$ $10^{-4}$ emu $\mathrm{Oe}^{-1} \mathrm{~mol}^{-1}$ for $B \perp c$, respectively.

\section{DISCUSSION}

Although not detectably hysteretic, the phase transition at $T_{p t} \approx 157 \mathrm{~K}$ in $\mathrm{Pr}_{4} \mathrm{Ni}_{3} \mathrm{O}_{10}$ is of first order, and must be slightly field dependent. This conclusion is supported by our combined heat-capacity, magnetic-susceptibility, and MR data as we will see in the following. The simplest explanation for the observed sign change of the MR around $T_{p t}$ (Fig. 4) is the assumption that the phase transition is shifted towards lower temperature with increasing magnetic field, thereby allowing the compound, once held at a temperature $T$ slightly below $T_{p t}$ in zero magnetic field, to re-enter the more metallic hightemperature phase with increasing field. From our resistance data (see Fig. 3) and assuming a parallel shift of $R(T)$ by $\Delta T_{p t}$ with increasing $B$, we estimate $\Delta T_{p t} \approx-0.1 \mathrm{~K}$ in $B=7 \mathrm{~T}$. Unfortunately, the temperature resolution of our heat-capacity data taken in zero magnetic field and in $B=7 \mathrm{~T}(\approx 0.5 \mathrm{~K})$ is not sufficient to resolve such a small effect of the magnetic field on $T_{p t}$ (see Fig. S1). However, the magnetic susceptibility exhibits a virtually field-independent steplike increase at $T_{p t}$ of the order of $\Delta \chi \approx 1.0 \times 10^{-4} \mathrm{emu} \mathrm{Oe}^{-1} \mathrm{~mol}^{-1}$ for $B \| c$ and $\Delta \chi \approx 3.7 \times 10^{-4} \mathrm{emu} \mathrm{Oe}^{-1} \mathrm{~mol}^{-1}$ for $B \perp c$, respectively [see Figs. 5(a), 5(b), and S5], indicating that the first-order phase transition at $T_{p t}$ as it is revealed by the heat capacity measurements must follow a nontrivial phase transition line $B_{p t}(T)$. If we relate the measured changes in magnetization at $T_{p t}, \Delta M=B \Delta \chi$, to the (apparently field-independent) entropy change $\Delta S \approx 2.0 \mathrm{~J} \mathrm{~mol}^{-1} \mathrm{~K}^{-1}$ via the Clausius-Clapeyron equation $-\Delta M\left(\frac{d B_{p t}}{d T}\right)=\Delta S$, which is valid at magnetic first-order transitions, we obtain $B_{p t}(T) \approx$ $\sqrt{2 \Delta S\left(T_{p t(B=0)}-T\right) / \Delta \chi}$, i.e., the phase-transition temperature must indeed decrease with increasing magnetic field $B$. This equation, together with the measured values for $\Delta \chi$ and $\Delta S$, predicts a decrease of $T_{p t}$ by $\Delta T_{p t} \approx-0.045 \mathrm{~K}$ in $B=7 \mathrm{~T}$ for $B \perp c$, which is in fair agreement with the above

TABLE II. Fitting results of the $\chi(T)$ data.

\begin{tabular}{|c|c|c|c|c|}
\hline & & $C\left(\mathrm{emu} \mathrm{K} \mathrm{Oe}{ }^{-1} \mathrm{~mol}^{-1}\right)$ & $\chi_{0}\left(\mathrm{emu} \mathrm{Oe}{ }^{-1} \mathrm{~mol}^{-1}\right)$ & $\Theta(\mathrm{K})$ \\
\hline Low-temperature phase & $\begin{array}{l}B \| c \\
B \perp c\end{array}$ & $\begin{array}{l}6.4(5) \\
6.4(1)\end{array}$ & $\begin{array}{l}3.9(9) \times 10^{-3} \\
2.2(7) \times 10^{-3}\end{array}$ & $\begin{array}{l}37 \pm 6 \\
35 \pm 1\end{array}$ \\
\hline
\end{tabular}


estimate from our MR data. It is, therefore, safe to conclude that the phase transition at $T_{p t}$, with its associated changes of the crystal structure and the physical properties, is field dependent and must be of first order, in agreement with Ref. [40].

We suggest that the very similar magnetoresistance effect observed at the spin-density-wave/charge-density-wave instability in $\mathrm{Na}_{2} \mathrm{Ti}_{2} \mathrm{Sb}_{2} \mathrm{O}$ around $T=110 \mathrm{~K}$ [66], can be explained in a similar fashion as being a consequence of a certain field dependence of this transition. We assume that such an effect is potentially also present in other systems showing field-dependent phase transitions that are accompanied by large resistance changes.

Although the metallicity is preserved for in-plane transport at all temperatures, the out-of-plane resistivity is enhanced below $T_{p t}$ [Fig. 3], which indicates either a metal-to-insulatorlike transition for the respective crystal direction, or at least a significant increase of the anisotropy between in-plane and out-of-plane properties induced by the structural phase transition at $T_{p t}$. With an average valance state of +2.67 , it is very likely that certain $E_{g}$ orbitals of the $\mathrm{Ni}$ ions are crossing the Fermi level, thereby forming partially filled energy bands. The situation is somewhat complicated by the fact that the inner $\mathrm{NiO}_{6}$ octahedra are contracted in the $c$ direction, while the outer ones are elongated (see Table I), leading to a reversed hierarchy of the respective $d_{x^{2}-y^{2}}$ and $d_{3 z^{2}-r^{2}}$ orbitals in energy for the two different $\mathrm{Ni}$ sites, and presumably resulting in a similarly complicated band structure as in $\mathrm{La}_{4} \mathrm{Ni}_{3} \mathrm{O}_{10}$ [24]. As the shape of $\mathrm{NiO}_{6}$ octahedra is altered at the phase transition, the associated changes in the electronic properties must be reflected in a corresponding reconstruction of the Fermi surface. The more pronounced change of the bond lengths within the inner $\mathrm{NiO}_{6}$ octahedra with decreasing temperature as compared to the outer ones may be due to a charge re-distribution between the inner and outer $\mathrm{NiO}_{6}$ octahedra, leading to possible charge and/or orbital order $[39,40]$ and an insulator-like behavior of the out-of-plane resistivity in the low-temperature phase.

The interpretation that the electronic structure undergoes a substantial change at $T_{p t}$ is supported by the fact that the Curie constant $C$ decreases when crossing $T_{p t}$ from above, and the Pauli-paramagnetic susceptibility as extracted from a Néeltype fit to the magnetic-susceptibility considerably increases (see Table II). This may indicate that the outer $3 d$ electrons of the Ni ions are essentially delocalized at low temperatures, while the increased value of $C$ in the high-temperature phase would be compatible with either three localized $S=1 / 2$ magnetic moments, or with one $\mathrm{Ni}$ ion in a high-spin $S=1$ configuration. It is worth noting here that the free-electron value for the Pauli-paramagnetic susceptibility for hypothetically $N=3$ mobile charge carriers per formula unit (one carrier per nickel atom) corresponds to $\chi_{0}^{\text {f.e }} \approx 10^{-4} \mathrm{emu} \mathrm{Oe}^{-1} \mathrm{~mol}^{-1}$, and varies only weakly with $N$ as $N^{1 / 3}$. As this value is compatible with the respective high-temperature values, the significant enhancement below the phase transition points to a substantial change of the electronic structure and a corresponding increase of the electronic density of states at the Fermi level with decreasing temperature.

\section{CONCLUSION}

To summarize, we have successfully grown single crystals of $\mathrm{Pr}_{4} \mathrm{Ni}_{3} \mathrm{O}_{10}$ with an optical-image floating-zone furnace at high oxygen pressure. We have performed comprehensive experiments in connection with the phase transition at $T_{p t} \approx 157 \mathrm{~K}$, such as single crystal XRD at different temperatures, heat capacity, electric-transport, and magnetization measurements. Whereas the resistivity data indicate a metalto-metal transition at $T_{p t}$ within the $a-b$ plane and a metal-toinsulator-like transition along the $c$ axis with decreasing temperature, the MR is enhanced right at $T_{p t}$ and exhibits a sign change, which we attribute to a slight suppression of the phase transition by a magnetic field. The first-order nature of this transition and its field dependence $T_{p t}(B)$ can be derived from the combined heat capacity, magnetic-susceptibility, and MR data. Both the resistance and magnetic-susceptibility measurements point to a reconstruction of the Fermi surface at $T_{p t}$, with a substantially enhanced Pauli-paramagnetic susceptibility in the more anisotropic low-temperature phase. These changes are likely related to a certain shape variation of the different $\mathrm{NiO}_{6}$ octahedra in the unit cell at $T_{p t}$ upon cooling from high to low temperatures.

\section{ACKNOWLEDGMENTS}

This work was supported by the Swiss National Science Foundation under Grants No. 20-175554, No. 206021163997, and No. PZ00P2-174015, and matching funds from the Paul Scherrer Institute for purchasing SCIDRE HKZ (the high-pressure, high-temperature optical-floating zone furnace).
[1] Y. Tokura and N. Nagaosa, Science 288, 462 (2000).

[2] L. de Medici, J. Mravlje, and A. Georges, Phys. Rev. Lett. 107, 256401 (2011).

[3] R. D. Shannon, D. B. Rogers, C. T. Prewitt, and J. L. Gillson, Inorg. Chem. 10, 4 (1971).

[4] S. Maekawa, T. Tohyama, S. E. Barnes, S. Ishihara, W. Koshibae, and G. Khaliullin, in Physics of Transition Metal Oxides, Vol.144 of Springer Series in Solid State Sciences (Springer-Verlag, Berlin, 2004).

[5] B. P. Stojkovic, Z. G. Yu, A. R. Bishop, A. H. Castro Neto, and N. Grønbech-Jensen, Phys. Rev. Lett. 82, 4679 (1999).

[6] T. Ito, H. Takagi, T. Ishibashi, and S. Uchida, Nature (London) 350, 596 (1991).
[7] C. N. R. Rao and B. Raveau, in Transition Metal Oxides (WileyVCH, New York, 1995).

[8] C. Renner, G. Aeppli, B. G. Kim, A. S. Yeong, and S. W. Cheong, Nature (London) 416, 518 (2002).

[9] J. G. Bednorz and K. A. Müller, Z. Phys. B 64, 189 (1986).

[10] B. Keimer, S. A. Kivelson, M. R. Norman, S. Uchida, and J. Zaanen, Nature (London) 518, 179 (2015).

[11] S. Jin, T. H. Tiefel, M. McCormack, R. A. Fastnacht, R. Ramesh, and L. H. Chen, Science 264, 413 (1994).

[12] M. Uehara, S. Mori, C. H. Chen, and S.-W. Cheong, Nature (London) 399, 560 (1999).

[13] J. Zaanen and O. Gunnarsson, Phys. Rev. B 40, 7391 (1989).

[14] S.-H. Lee and S.-W. Cheong, Phys. Rev. Lett. 79, 2514 (1997). 
[15] N. F. Mott and L. Friedman, Philos. Mag. 30, 389 (1974).

[16] J. B. Torrance, P. Lacore, A. I. Nazzal, E. J. Ansaldo, and Ch. Niedermayer, Phys. Rev. B 45, 8209(R) (1992).

[17] X. Obradors, L. M. Paulius, M. B. Maple, J. B. Torrance, A. I. Nazzal, J. Fontcuberta, and X. Granados, Phys. Rev. B 47, 12353 (1993).

[18] P. C. Canfield, J. D. Thompson, S-W. Cheong, and L. W. Rupp, Phys. Rev. B 47, 12357 (1993).

[19] J. Shamblin, M. Heres, H. Zhou, J. Sangoro, M. Lang, J. Neuefeind, J. A. Alonso, and S. Johnston, Nat. Commun. 9, 86 (2018).

[20] P. A. Lee, N. Nagaosa, and X.-G. Wen, Rev. Mod. Phys. 78, 17 (2006).

[21] Y. Kohsaka, T. Hanaguri, M. Azuma, M. Takano, J. C. Davis, and H. Takagi, Nat. Phys. 8, 534 (2012).

[22] J. Chaloupka and G. Khaliullin, Phys. Rev. Lett. 100, 016404 (2008).

[23] V. V. Poltavets, K. A. Lokshin, A. H. Nevidomskyy, M. Croft, T. A. Tyson, J. Hadermann, G. V. Tendeloo, T. Egami, G. Kotliar, N. ApRoberts-Warren, A. P. Dioguardi, N. J. Curro, and M. Greenblatt, Phys. Rev. Lett. 104, 206403 (2010).

[24] H. Li, X. Zhou, T. Nummy, J. Zhang, V. Pardo, W. E. Pickett, J. F. Mitchell, and D. S. Dessau, Nat. Commun. 8, 704 (2017).

[25] J. Zhang, A. S. Botana, J. W. Freeland, D. Phelan, H. Zheng, V. Pardo, M. R. Norman, and J. F. Mitchell, Nat. Phys. 13, 864 (2017)

[26] D. Li, K. Lee, B. Y. Wang, M. Osada, S. Crossley, H. R. Lee, Y. Cui, Y. Hikita, and H. Y. Hwang, Nature (London) 572, 624 (2019).

[27] P. Lacorre, J. B. Torrance, J. Pannetier, A. I. Nazzal, P. W. Wang, and T. C. Huang, J. Solid State Chem. 91, 225 (1991).

[28] G. Catalan, Phase Trans. 81, 729 (2008).

[29] D. J. Gawryluk, Y. M. Klein, T. Shang, D. Sheptyakov, L. Keller, N. Casati, Ph. Lacorre, M. T. Fernández-Díaz, J. Rodríguez-Carvajal, and M. Medarde, Phys. Rev. B 100, 205137 (2019).

[30] J. A. Alonso, M. J. Martinez-Lope, M. T. Casais, M. A. G. Aranda, and M. T. Fernandez-Diaz, J. Am. Chem. Soc. 121, 4754 (1999).

[31] S. Johnston, A. Mukherjee, I. Elfimov, M. Berciu, and G. A. Sawatzky, Phys. Rev. Lett. 112, 106404 (2014).

[32] A. Subedi, O. E. Peil, and A. Georges, Phys. Rev. B 91, 075128 (2015).

[33] V. Bisogni, S. Catalano, R. J. Green, M. Gibert, R. Scherwitzl, Y. Huang, V. N. Strocov, P. Zubko, S. Balandeh, J.-M. Triscone, G. A. Sawatzky, and T. Schmitt, Nat. Commun. 7, 13017 (2016).

[34] J. Rodriguez-Carvajal, J. L. Martinez, J. Pannetier, and R. Saez-Puche, Phys. Rev. B 38, 7148 (1988).

[35] M. T. Fernandez-Diaz, J. Rogriguez-Carvajal, J. L. Martinez, G. Fillion, F. Fernandez, and R. Saez-Puche, Z. Phys. B 82, 275 (1991).

[36] J. Rodriguez-Carvajal, M. T. Fernandez, J. L. Martinez, F. Fernandez, and R. Saez-Puche, Europhys. Lett. 11, 261 (1990).

[37] S. R. Maity, M. Ceretti, L. Keller, J. Schefer, T. Shang, E. Pomjakushina, M. Meven, D. Sheptyakov, A. Cervellino, and W. Paulus, Phys. Rev. Mater. 3, 083604 (2019).
[38] H. Sasaki, H. Harashina, S. Taniguchi, M. Kasai, Y. Kobayashi, M. Sato, T. Kobayashi, T. Ikeda, M. Takata, and M. Sakata, J. Phys. Soc. Jpn. 66, 1693 (1997).

[39] G. Wu, J. J. Neumeier, and M. F. Hundley, Phys. Rev. B 63, 245120 (2001).

[40] Z. Zhang and M. Greenblatt, J. Solid State Chem. 117, 236 (1995).

[41] S. Kumar, Ø. Fjellvåg, A. O. Sjåstad, and H. Fjellvåg, J. Magn. Magn. Mater. 496, 165915 (2020).

[42] M. Greenblatt, Curr. Opin. Solid State Mater. Sci. 2, 174 (1997).

[43] V. V. Poltavets, K. A. Loshkin, T. Egami, and M. Greenblatt, Mater. Res. Bull. 41, 955 (2006).

[44] D. Das, M. Pal, E. Di-Bartolomeo, E. Traversa, and D. Chakravorty, J. Appl. Phys. 88, 6856 (2000).

[45] J. Zhang, H. Zheng, Y. Ren, and J. F. Mitchell, Crystallogr. Growth Des. 17, 2730 (2017).

[46] H. Guo, Z. W. Li, L. Zhao, Z. Hu, C. F. Chang, C.-Y. Kuo, W. Schmidt, A. Piovano, T. W. Pi, O. Sobolev, D. I. Khomskii, L. H. Tjeng, and A. C. Komarek, Nat. Commun. 9, 43 (2018).

[47] D. J. Buttrey, H. R. Harrison, J. M. Honig, and R. R. Schartman, J. Solid State Chem. 54, 407 (1984).

[48] J.M. Bassat, F. Gervais, P. Odier, and J. P. Loup, Mater. Sci. Eng. B 3, 507 (1989).

[49] J. Zhang, H. Zheng, Y. S. Chen, Y. Ren, M. Yonemura, A. Huq, and J. F. Mitchell, arXiv:1904.10048 (2019).

[50] M. D. Carvalho, F. M. A. Costa, Isabelda S. Pereira, A. Wattiaux, J. M. Bassat, J. C. Grenier, and M. Pouchard, J. Mater. Chem. 7, 2107 (1997).

[51] See Supplemental Material at http://link.aps.org/supplemental/ 10.1103/PhysRevB.101.104104 for more details about experimental methods, crystallographic data, the heat capacity in an external magnetic field, and analysis of the magnetic data.

[52] H. M. Rietveld, J. Appl. Crystallogr. 2, 65 (1969).

[53] J. Rodríguez-Carvajal, Physica B 192, 55 (1993).

[54] T. Roisnel and J. Rodriguez-Carvajal, in Proceedings of Seventh European Powder Diffraction Conference (EPDIC 7), edited by R. Delhez and E. J. Mittenmeijer (Trans Tech Publications Ltd., Switzerland, 2000), pp. 118.

[55] Rigaku Oxford Diffraction, https://www.rigaku.co.jp/, 2015.

[56] R. C. Clark and J. S. Reid, Acta Crystallogr. A 51, 887 (1995).

[57] CrysAlisPro (version 1.171.40.53), Rigaku Oxford Diffraction, Japan, 2019.

[58] O. V. Dolomanov, L. J. Bourhis, R. J. Gildea, J. A. K. Howard, and H. Puschmann, J. Appl. Crystallogr. 42, 339 (2009).

[59] G. M. Sheldrick, Acta Crystallogr. A71, 3 (2015).

[60] R. F. Jardim, V. B. Barbeta, S. Andrade, M. T. Escote, F. Cordero, and M. S. Torikachvili, J. Appl. Phys. 117, 17C105 (2015).

[61] G. Bergmann, Phys. Rep. 107, 1 (1984).

[62] D. V. Baxter, R. Richter, M. L. Trudeau, R. W. Cochrane, and J. O. Strom-Olsen, J. Phys. (France) 50, 1673 (1989).

[63] M. D. Carvalho, M. M. Cruz, A. Wattiaux, J. M. Bassat, F. M. A. Costa, and M. Godinho, J. Appl. Phys. 88, 544 (2000).

[64] X. Q. Xu, J. L. Peng, Z. Y. Li, H. L. Ju, and R. L. Greene, Phys. Rev. B 48, 1112 (1993).

[65] R. Saez-Puche, F. Fernandez, J. Rodriguez-Carvajal, and J. L. Martinez, Solid State Commun. 72, 273 (1989).

[66] R. H. Liu, D. Tan, Y. A. Song, Q. J. Li, Y. J. Yan, J. J. Ying, Y. L. Xie, X. F. Wang, and X. H. Chen, Phys. Rev. B 80, 144516 (2009). 